\title{
Mielotoxicidad inducida por estrógenos de origen ovárico y leiomiosarcoma vulvar en una perra. Comunicación de caso
}

\section{Ovarian oestrogen-induced myelotoxicity and vulvar leiomyosarcoma in a bitch. Case report}

\author{
Osorio-Baños Julio Cesar ${ }^{1,2,0 *}$, Servín Trujillo Miguel Ángel ${ }^{3}$, \\ GARRIDo Becerril Ingrid Patricia 3
}

1. Unidad de Posgrado, Facultad de Medicina Veterinaria y Zootecnia, Universidad Nacional Autónoma de México. 2. Laboratorio de Cáncer Pulmonar, Instituto Nacional de Enfermedades Respiratorias "Ismael Cosío Villegas”, México. 3. Centro Médico Veterinario de Tulancingo, Hidalgo. México.

* Correo electrónico del autor de contacto:juliocesarosba@hotmail.com

\begin{abstract}
Resumen
Las elevadas concentraciones sanguíneas de estrógenos resultan tóxicas para la médula ósea, contribuyendo al desarrollo de hipoplasia medular severa. En estados crónicos, esta puede ser irreversible. Los estrógenos influyen en el desarrollo de tumores del músculo liso en útero, vagina y vulva. Los sarcomas primarios representan solamente el 1-3 \% de las neoplasias malignas vulvares en perras; el leiomiosarcoma es el más común. En este trabajo se describe un caso de mielotoxicidad inducida por estrógenos de origen ovárico y un leiomiosarcoma vulvar en una perra pit bull de 7 años. El animal presentaba hiporexia, depresión, dificultad para desplazarse, disnea, dolor a la palpación en la región inguinal, disuria, edema vulvar, alopecia simétrica bilateral, agrandamiento de glándulas mamarias y ciclos reproductivos anormales, así como una masa localizada en el labio vulvar izquierdo. Mediante estudios de laboratorio se demostró pancitopenia severa asociada a mielotoxicidad y una concentración de $17 \beta$-estradiol sérico de $185,6 \mathrm{pg} / \mathrm{ml}$. Mediante el examen citológico de la médula ósea se determinó hipoplasia medular. Se realizaron escisión quirúrgica de la masa tumoral y ooforosalpingectomía, encontrándose un quiste folicular en el ovario derecho. Se realizaron estudios histopatológicos e inmunohistoquímicos y se formuló un diagnóstico definitivo de mielotoxicidad inducida por estrógenos y leiomiosarcoma vulvar.
\end{abstract}

\section{Palabras clave}

anemia, estrógenos, leiomiosarcoma vulvar, inmunohistoquímica

\begin{abstract}
High blood oestrogen concentration leads to bone marrow toxicity contributing to the development of severe and sometimes irreversible bone marrow hypoplasia. Oestrogens also play a role in the development of smooth muscle tumors in the uterus, vagina and vulva. Primary sarcomas represent only 1-3 \% of malignant vulvar neoplasms in dogs, with leiomyosarcoma being the most common. This report describes a case of myelotoxicity and vulvar leiomyosarcoma due to ovarian hyperoestrogenism in a 7year-old pit bull. The patient came to the clinic presenting hyporexia, depression, difficulty to move, dyspnea, pain upon inguinal palpation, dysuria, vulvar edema, bilateral symmetric alopecia, growth of mammary glands and abnormal reproductive cycles, as well as a mass located in the left vulvar lip. Laboratory studies showed severe pancytopenia associated with myelotoxicity and a serum $17 \beta$-oestradiol concentration of $185.6 \mathrm{pg} / \mathrm{ml}$. Hypoplasia was found in the bone marrow aspirate. Surgical excision of the mass was carried out together with oophorosalpingectomy, and a follicular cyst was found in the right ovary. Histopathological and immunohistochemical studies were carried out and a definitive diagnosis of oestrogen-induced myelotoxicity and vulvar leiomyosarcoma was made.
\end{abstract}

\section{Key words}

anaemia, oestrogen, vulvar leiomyosarcoma, immunohistochemistry

Fecha de recepción: 01/04/2018

Analecta Vet 2018; Julio-Diciembre; 38(2):32-37

Impresa ISSN 03655 14-8 Electrónica ISSN 1514-2590

Fecha de aprobación: 21/08/2018

doi.org/10.24215/15142590eo3o 


\section{Introducción}

Los estrógenos son compuestos esteroides sintetizados principalmente en los ovarios, corteza adrenal y, en menor cantidad, en hígado, músculos y tejido adiposo (Sontas et al., 2009). Los estrógenos son esenciales para el crecimiento, la formación de características sexuales secunda-rias, tales como el desarrollo de glándulas mama-rias, los cambios conformacionales, el comporta-miento y el mantenimiento del ciclo estral; durante el estro y proestro existe mayor concentración de estrógenos séricos (Almeida et al., 2017). Los estrógenos reducen la hematopoyesis por toxicidad directa sobre las células progenitoras y alteran el microambiente estromal disminuyendo los factores de crecimiento e inhibiendo la capacidad de proliferación sobre la médula ósea (Sanpera et al., 2002; Weiss \& Wardrop, 2010). En las primeras fases de este proceso se observa hiperplasia granulocítica con leucocitosis, neutrofilia y desviación a la izquierda, trombocitopenia y anemia. En etapas avanzadas, se detecta una pancitopenia moderada a severa con hipoplasia o aplasia medular (Ishii \& Young, 2015). Algunas pacientes se recuperan una vez eliminado el agente causal. En estadios crónicos se desarrolla una aplasia medular irreversible con anemia, hipoxemia sistémica, hemorragias masivas e infecciones bacterianas secundarias. Todo esto conduce a un cuadro de shock séptico (Reagan et al., 2011). El incremento de estrógenos puede favorecer el desarrollo de neoplasias de músculo liso en cualquier sitio del tracto reproductor (D'Angelo \& Prat, 2009; Puie, 2017).

El leiomiosarcoma es una neoplasia mesenquimática maligna de tejidos blandos. En medicina humana representa aproximadamente el 1-3 \% de todos los tumores vulvares malignos (Ávila et al., 2001; Suh, 2008). En perras, los tumores uterinos y vulvares representan el o,4 \% de todas las neoplasias; entre ellos, el $10 \%$ son leiomiosarcomas (Serin et al., 2010). En mujeres, estas neoplasias se localizan principalmente en los labios mayores, pero también se han reportado en clítoris y labios menores (Teramae et al., 2014); en perras las paredes vaginales y vestibulares son las más afectadas, mientras que el clítoris y los labios vulvares son las zonas menos involucradas (Ranieri et al., 2018).

Existen escasas comunicaciones acerca de este tumor en animales domésticos, por lo que no se ha establecido la prevalencia según edad ni raza (Manothaludum et al., 1991; Ranieri et al., 2018; Sontas, 2009). Debido a su rareza, tanto en medicina humana como en veterinaria, los protocolos de tratamiento no se encuentran disponibles. Por lo tanto, la escisión con bordes quirúrgicos amplios es el tratamiento recomendado (Behranwala et al., 2004; Çobanoğlu et al., 1996; Moravek et al., 2015; Teramae et al., 2014).

El objetivo de esta comunicación es describir un caso de mielotoxicidad por hiperestro- genismo ovárico y un leiomiosarcoma vulvar en una perra.

\section{Presentación del caso}

Se presentó a la consulta una perra pit bull, de 7 años, no castrada, con antecedentes de hiporexia, depresión, dificultad para desplazarse, disnea, ninfomanía y secreción vaginal serosanguinolenta de tres semanas de duración. Durante el examen clínico se observaron las mucosas pálidas, alopecia simétrica bilateral con marcada hiperpigmentación cutánea en la región inguinal, agrandamiento de las glándulas mamarias, abdomen distendido con dolor a la palpación, disuria, descarga vulvar sanguinolenta y hemorragias petequiales y equimóticas en el abdomen y dorso. En el labio vulvar izquierdo se apreció una masa bien delimitada, de $25 \mathrm{~cm}$ de diámetro, ulcerada y firme al tacto. Se obtuvieron muestras de sangre para hemograma y química sanguínea y de orina para urianálisis, así como una radiografia latero-lateral de la lesión.

Tabla 1. Hemograma

\begin{tabular}{llll}
\hline Analito & Resultado & Rango de referencia & Unidades \\
\hline Hematocrito & 0,19 & $0,37-0,55$ & $\mathrm{l} / 1$ \\
Hemoglobina & 54 & $120-180$ & $\mathrm{~g} / 1$ \\
Eritrocitos & 3,1 & $5,5-8,5$ & $\mathrm{\times} 10^{12} / 1$ \\
Volumen globular medio & 61,2 & $60-77$ & $\mathrm{fl}$ \\
$\begin{array}{l}\text { Concentración globular media de } \\
\text { hemoglobina }\end{array}$ & 284,2 & $320-360$ & $\mathrm{~g} / 1$ \\
Reticulocitos & & & \\
Plaquetas & 0 & $<60$ & $\mathrm{x} 10^{9 / 1}$ \\
Sólidos totales & 105 & $200-900$ & $\mathrm{x} 10^{9 / 1}$ \\
Leucocitos & 55 & $60-75$ & $\mathrm{~g} / 1$ \\
Neutrófilos & 3,3 & $6,0-17,0$ & $\times 10^{9 / 1}$ \\
Neutrófilos en banda & 2,3 & $3,0-11,1$ & $\times 10^{9 / 1}$ \\
Linfocitos & 0 & $0-0,3$ & $\mathrm{x} 10^{9 / 1}$ \\
Monocitos & 0,6 & $1,0-4,8$ & $\mathrm{x} 10^{9 / 1}$ \\
Eosinófilos & 0,3 & $0,1-1,4$ & $\mathrm{x} 10^{9 / 1}$ \\
Basófilos & 0,1 & $0,1-0,9$ & $\times 10^{9 / 1}$ \\
Tiempo de protrombina & 0 & $0-0,2$ & $\mathrm{x} 10^{9 / 1}$ \\
Tiempo de trombina & 8 & $6-12$ & $\mathrm{~s}$ \\
Tiempo de tromboplastina parcial & 5 & $3-8$ & $\mathrm{~s}$ \\
activada & 14 & $11-17$ & $\mathrm{~s}$ \\
\hline
\end{tabular}

El hemograma reveló anemia normocítica hipocrómica no regenerativa severa, que se asoció con daño medular y hemorragia, leucopenia por neutropenia, linfopenia y trombocitopenia atribuidas a hipoplasia medular (Tabla 1). Los hallazgos en las determinaciones bioquímicas incluyeron hiperbilirrubinemia e incremento de fosfatasa alcalina (relacionadas con colestasis), hipoproteinemia por albuminemia e hipoglucemia (que se asociaron con la hiporexia), incremento de alanina aminotransferasa y aspartato aminotransferasa debido a degeneración hepatocelular, e hiperazotemia por compromiso renal (Tabla 2). En las pruebas de coagulación el tiempo de protrombina, trombina y el tiempo de tromboplastina parcial activada fueron normales. En la placa radiográfica se observó una masa radiopaca, bien delimitada con densidad homogénea (Figura 1). 
Tabla 2. Bioquímica sanguínea

\begin{tabular}{llll}
\hline Analito & Resultado & Rango de referencia & Unidades \\
\hline Proteinas totales & 55,2 & $56,6-74,8$ & $\mathrm{~g} / 1$ \\
Albúmina & 24,7 & $29-40$ & $\mathrm{~g} / 1$ \\
Globulinas & 30,5 & $24-39$ & $\mathrm{~g} /$ \\
Relación A/G & 0,80 & $0,78-1,46$ & \\
Glucosa & 2,03 & $3,3-6,8$ & $\mathrm{mmol} / 1$ \\
Urea & 14,2 & $2,1-7,9$ & $\mathrm{mmol} / 1$ \\
Creatinina & 169,4 & $60-126$ & $\mu \mathrm{mol} / 1$ \\
Bilirrubina total & 8,2 & $<5,2$ & $\mu \mathrm{mol} / 1$ \\
Bilirrubina directa & 3,1 & $<1,0$ & $\mu \mathrm{mol} / 1$ \\
Bilirubina indirecta & 5,1 & $<5,0$ & $\mu \mathrm{mol} / 1$ \\
Alanina aminotransferasa & 113,8 & $4-70$ & $\mathrm{UI} / 1$ \\
Aspartato aminotransferasa & 84,2 & $12-55$ & $\mathrm{UI} / 1$ \\
Fosfatasa alcalina & 204,7 & $6-189$ & $\mathrm{UI} / 1$ \\
Creatina cinasa & 102,8 & $17-213$ & $\mathrm{UI} /$ \\
\hline
\end{tabular}

Sobre la base de los resultados de laboratorio se realizó aspirado de médula ósea en la cresta iliaca derecha. Los extendidos fueron fijados y teñidos con la técnica de Wright, observándose marcada disminución de las líneas eritrocítica, mielocítica y linfocítica confirmando el diagnóstico de hipoplasia medular (Figura 2). Se tomaron varias muestras por aspirado de la masa vulvar con aguja delgada, las que fueron fijadas en alcohol $96^{\circ}$ y teñidas mediante la técnica de Papanicolaou. Se apreció un fondo de aspecto proteináceo con abundantes eritrocitos y numerosas células fusiformes neoplásicas dispuestas en forma aislada o en grupos. Estas presentaban bordes celulares parcialmente definidos, citoplasma pálido y núcleo central y oval de extremos romos, de cromatina fina granular, con uno o dos nucléolos evidentes. Se apreciaron moderada anisocitosis, anisocariosis y cariomegalia, incremento de la relación núcleo-citoplasma y pleomorfismo celular (Figura 3).

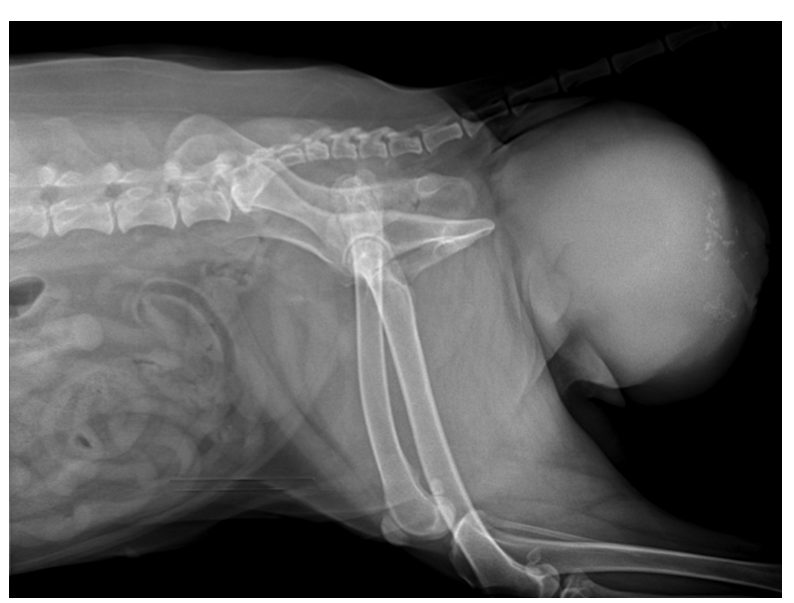

Figura 1. Masa vulvar, bien delimitada, radiolúcida, con densidad homogénea. Proyección latero-lateral.

El valor sérico del $17 \beta$-estradiol fue de $185,6 \mathrm{pg} / \mathrm{ml}$ (rango de referencia $50-80 \mathrm{pg} / \mathrm{ml}$ ). Sobre la base de la historia clínica, la evaluación física y los resultados de laboratorio se confirmó una mielotoxicidad inducida por estrógenos.
Se realizó la escisión total de la masa tumoral con vulvectomía radical y bordes quirúrgicos amplios (Figura 4), y ooforosalpingectomía, constatándose un quiste folicular de $6,5 \mathrm{~cm}$ de diámetro en el ovario derecho. En las muestras de la masa tumoral, procesadas mediante técnicas de rutina, se observó un tejido de neoformación bien delimitado, encapsulado, compuesto por numerosas células mesenquimáticas neoplásicas dispuestas en haces cortos entrelazados o en patrón verticilar, delimitadas y sostenidas por un fino estroma fibrovascular. En algunas zonas se apreciaron extensas áreas de necrosis coagulativa y hemorragias. Se contaron 19 figuras mitóticas en 10 campos aleatorios con el objetivo de 40x. Se realizó inmunocitoquímica indirecta mediante la técnica de estreptoavidina-biotina-peroxidasa. Las muestras resultaron positivas para vimentina, desmina y actina de músculo liso. Los resultados fueron negativos para factor VIII y mioglobina. Se formuló el diagnóstico definitivo de leiomiosarcoma vulvar (Figura 5).

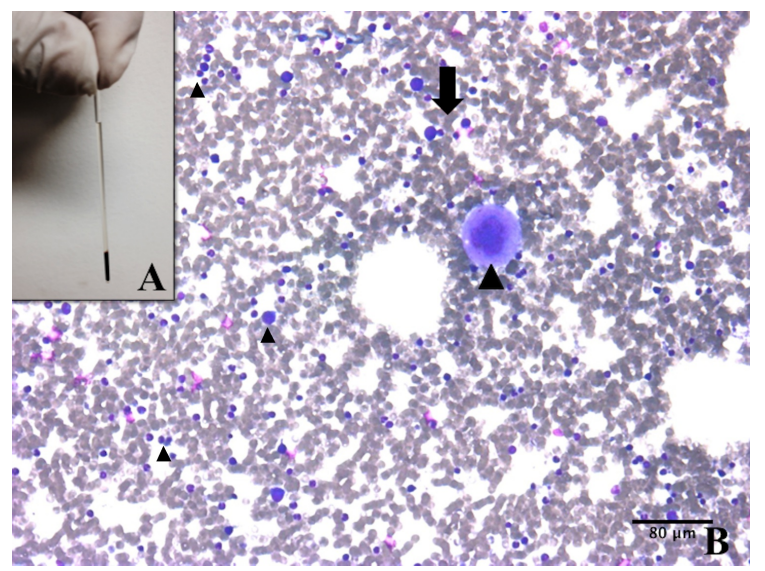

Figura 2. (A). Marcada disminución del hematocrito -anemia severa- (o,19 Hto) (B). Aspirado de médula ósea en la que se aprecia marcada disminución de las líneas eritrocítica (flecha) y mielocítica (puntas de flecha). Wright. Barra $=80 \mu \mathrm{m}$.

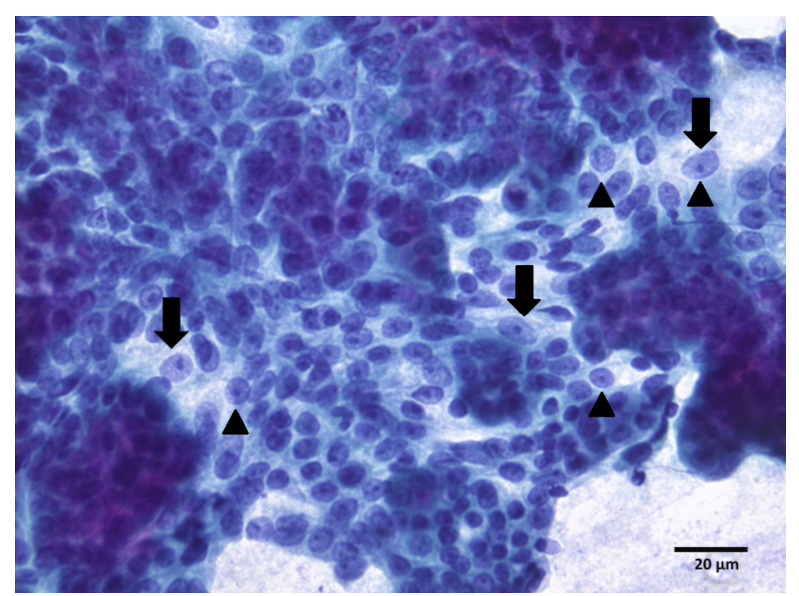

Figura 3. Grupos de células fusiformes con bordes parcialmente definidos (flechas), núcleo central oval con cromatina finamente granular y nucléolo prominente (puntas de flecha). Técnica de Papanicolaou. Barra $=20 \mu \mathrm{m}$. 


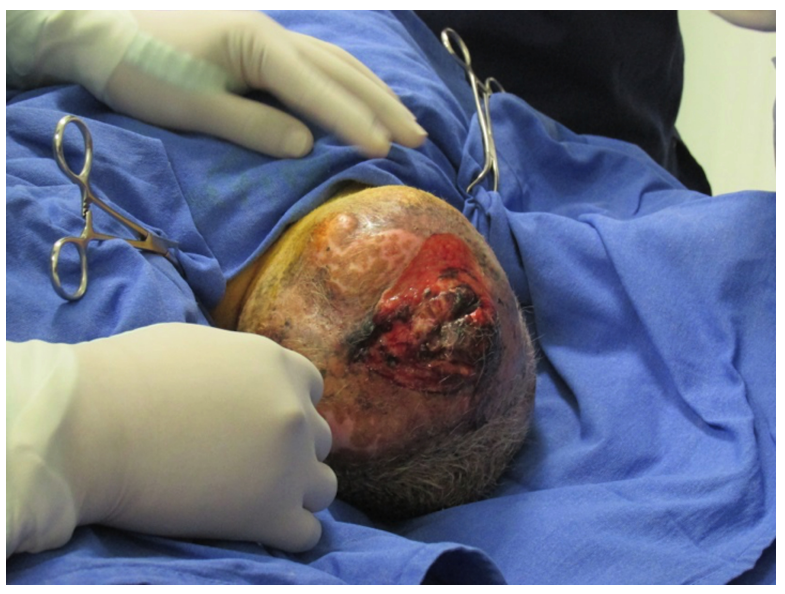

Figura 4. Escisión quirúrgica de la masa, encapsulada y de superficie multinodular con bordes quirúrgicos amplios.

Luego de la cirugía, se instauró un tratamiento con doxiciclina con dosis de $10 \mathrm{mg} / \mathrm{kg}$ cada 24 horas, por vía oral, durante 10 días. También se practicó una transfusión sanguínea a fin de restaurar los valores de eritrocitos y plaquetas. Previamente se realizaron pruebas cruzadas de compatibilidad con un perro donante clínicamente sano. Tres semanas después de la cirugía se tomaron muestras sanguíneas para hemograma y química sanguínea, en las cuales se observó ligera leucopenia por neutropenia y un valor de $17 \beta$-estradiol sérico de
$65 \mathrm{pg} / \mathrm{ml}$. Seis meses después, cuando se evaluó a la paciente, no presentaba ninguna alteración hematológica ni evidencia de recurrencia tumoral (Figura 6).

\section{Discusión}

La mielotoxicidad inducida por estrógenos genera un daño directo en las células precursoras hematopoyéticas de la médula ósea, induciendo un aumento transitorio en la granulopoyesis. En el hemograma se observan leucocitosis por neutrofilia y anemia normocítica normocrómica no regenerativa asociadas a hipoplasia medular, la que provoca pancitopenia de moderada a grave. Las petequias, la secreción serosanguinolenta y el edema vulvar observados en la paciente sugieren un efecto estrogénico (Pineda et al., 2008; Reagan et al., 2011; Ishii \& Young, 2015). En casos de exposición crónica, la pancitopenia puede persistir, a pesar de la eliminación de la fuente de estrógenos, siendo irreversible (Weiss et al., 2010; Almeida et al., 2017). Las pacientes son susceptibles a infecciones bacterianas debido a la neutropenia, trombocitopenia y anemia, por lo que se requieren un esquema de tratamiento con antibioterapia de amplio espectro y la restauración del volumen sanguíneo circulante. La sensibilidad de la médula ósea a los efectos mielotóxicos de los

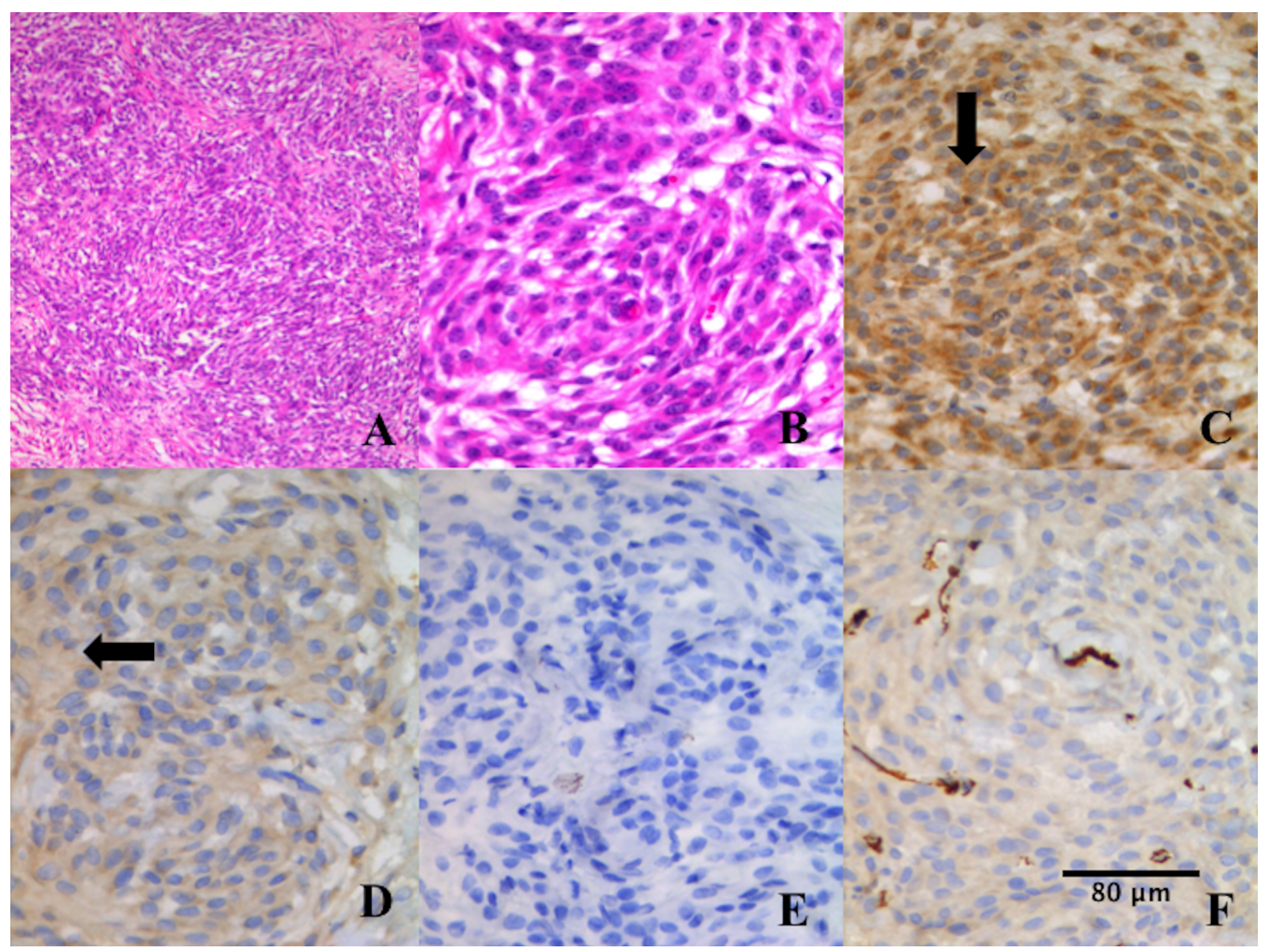

Figura 5. Aspecto microscópico e inmunotipificación del tumor. (A). Células neoplásicas en un patrón verticilar formando haces. HE. (B). Células fusiformes con bordes poco definidos y cromatina finamente granular. HE. (C). Actina de músculo liso, positividad en citoplasma (flecha). IHQ. (D). Vimentina, positividad en citoplasma (flecha). IHQ (E). Mioglobina, IHQ. (F). Desmina. IHQ. Barra = (A) $160 \mu \mathrm{m}$; (B-F) $80 \mu \mathrm{m}$. 
estrógenos depende de muchos factores, incluyendo la edad, la condición física y el estado de nutrición del paciente. Los gatos presentan una fuerte resistencia a la toxicidad de estrógenos, mientras que los perros son muy sensibles (Sontas et al., 2009).

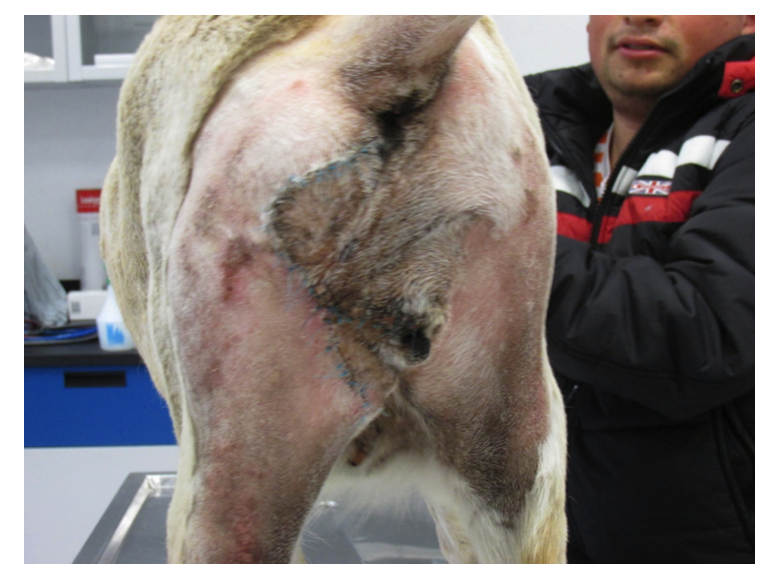

Figura 6. Evaluación posoperatoria a 6 meses, sin recidiva tumoral.

El leiomiosarcoma vulvar es una neoplasia poco frecuente en animales domésticos, es de comportamiento localmente infiltrativo a tejidos blandos adyacentes y se origina de células mesenquimáticas con diferenciación muscular lisa (Teramae et al., 2014). Entre los principales diagnósticos diferenciales que se deben considerar se encuentran el rabdomiosarcoma, el fibrosarcoma, el fibroma, el fibrohistiocitoma, el hemangiosarcoma, el mixosarcoma, el liposarcoma e inclusive los quistes de las glándulas vestibulares mayores (en humanos) (Korkmaz et al., 2016; Manothaludum, 1991). La inmunotipificación se ha convertido en una técnica complementaria del diagnóstico morfopatológico de tumores y cada vez ha cobrado mayor importancia en medicina veterinaria. Su implementación tiene valor diagnóstico, pronóstico y de orientación terapéutica (Ramos-Vara, 2005). D'Angelo \& Prat, (2009) comunican que desde el punto de vista inmunocitoquímico, los leiomiosarcomas muestran reacción positiva para la actina de músculo liso (SMA), desmina y vimentina, tal como ocurrió en este caso, y negativa para la proteína $\mathrm{S} 100$, mioglobina, FVIII y CD34 (Dennis et al., 2011).

El $85 \%$ de los tumores de músculo liso de los sistemas urinario y reproductor en caninos se localiza en el útero. Estos tumores son, en su mayor parte, benignos (leiomiomas) y están asociados con un desequilibrio hormonal (Çobanoğlu et al., 1996). Los leiomiosarcomas, además de en el útero, han sido hallados en la vagina, uretra $y$, rara vez, en ovarios, ligamento úterosacro, retroperitoneo y vulva, tanto en mujeres como en perras (Ávila et al., 2001; Ranieri et al., 2018; Suh \& Park, 2008). En este caso el tumor se localizó en el labio vulvar izquierdo, un sitio poco frecuente. El incremento de estrógenos de origen ovárico produ- jo alopecía; también se observó el agrandamiento de las glándulas mamarias y cambios de comportamiento como ninfomanía. La alta concentración de estrógenos circulantes causó toxicidad en las células hematopoyéticas originando pancitopenia.

Los escasos reportes registrados acerca del leiomiosarcoma vulvar indican que suele localizarse en los labios mayores, que es un tumor único, redondeado, bien delimitado y encapsulado, como se observó en este caso. La causa no está establecida; sin embargo, Teramae et al. (2014) informan que el leiomiosarcoma aparece durante la etapa reproductiva; en este caso se sugiere la participación del incremento de estrógenos de origen ovárico como factor predisponente en el desarrollo del leiomiosarcoma (Meuten, 2016, Teramae et al., 2014). Dennis et al. (2011) describen varios factores predictivos y pronósticos entre los cuales incluyen el grado histológico, el tamaño del tumor, su localización, la invasión a tejidos adyacentes y el índice mitótico, éste último es un factor pronóstico y diagnóstico; un alto índice mitótico se asocia con recurrencia, metástasis y reducción en el tiempo de sobrevida.

Sanpera et al. (2002), en la descripción de un caso, concluyen que la interrupción en la producción de estrógenos o de progesterona de origen ovárico por agonistas de GnRH resulta en la reducción del tamaño del tumor. Sin embargo, el tratamiento establecido es la escición quirúrgica del tumor con bordes quirúrgicos amplios.

\section{Conclusiones}

El leiomiosarcoma vulvar debe ser considerado entre los diagnósticos diferenciales frente a una masa vulvar en una perra. En casos de anemia concomitante se recomienda la medición de la concentración de estradiol. El diagnóstico definitivo del tumor se basa en sus características morfológicas y el factor pronóstico más relevante es el grado de diferenciación del tumor. La incorporación de la inmunohistoquímica en el diagnóstico de tumores vulvares ha sido útil para facilitar el diagnóstico asertivo.

\section{Agradecimientos}

Los autores agradecen al Hospital Veterinario de la Universidad Autónoma del Estado de Hidalgo-México por el apoyo en la utilización del equipo para la obtención de las radiografias digitales y en el seguimiento del caso.

\section{Conflicto de intereses}

Los autores declaran que no existe conflicto de intereses, incluyendo las relaciones financieras, personales o de otro tipo con otras personas u organizaciones que pudieran influir de manera inapropiada en el trabajo. 


\section{Bibliografía}

Almeida M, Laurent MR, Dubois V, Claessens F, O’Brien A, Bouillon R, Vanderschueren D. 2017. Estrogens and androgens in skeletal physiology and pathophysiology. Physiological Reviews. 97(1): 135-87. doi: 10.1152/physrev.00033.2015

Ávila ML, Torres AL, Cruz OH, Rojo HG. 2001. Leiomiosarcoma de la vulva. Presentación de un caso con 22 años de seguimiento. Revista Médica del Hospital General de México. 64(2):100-3.

Behranwala KA, Latifaj B, Blake P, Barton DPJ, Shepherd JH, Thomas JM. 2004. Vulvar soft tissue tumors. International Journal of Gynecological Cancer. 14(1):94-9.

Çobanoğlu Ö, Gürkan ZC, Ergun Y, Kutivay L. 1996. Leiomyosarcoma of the vagina. European Journal of Obstetries \& Gynecology and Reproductive Biology. 70(2):205-207.

D'Angelo E, Prat J. 2009. Uterine sarcomas: a review. Gynecologic Oncology. 30(1):1-9.

doi: 10.1016/j.ygyno.2009.09.023

Dennis MM, McSporran KD, Bacon NJ, Schulman FY, Foster RA, Powers BE. 2011. Prognostic factors for cutaneous and subcutaneous soft tissue sarcomas in dos. Veterinary Pathology. 48(1):7384. doi: 10.1177/0300985810388820

Ishii K, Young NS. 2015. Anemia of central origin. Seminars in Hematology. 52 (4):321-38.

doi: 10.1053/j.seminhematol.2015.07.002

Korkmaz V, Kurdoglu Z, Karadag B, Arslanca T, Caydere M, Ergun Y. 2016. A rare case of leiomyosarcoma localized in the Bartholin's gland area and review of the literature. Journal of Obstetrics and Gynaecology Research. 42(1):58992. doi: 10.1111/jog.12943

Manothaludum K, Johnston SD. 1991. Clinical approach to vaginal/vestibular masses in the bitch. Veterinary Clinics of North America: Small Animal Practice. 21 (3):509-21.

Meuten DJ. 2016. Tumors in domestic animals. $5^{\mathrm{O}}$ Ed. John Wiley \& Sons Inc. Iowa.

Moravek MB, Yin P, Ono Masanori, Coon JSV, Dyson MT, Navarro A, Marsh EE. 2015. Ovarian steroids, stem cells and uterine leiomyoma: therapeutic implications. Human Reproduction Update. 21(1):1-12.

doi: 10.1093/humupd/dmuo48
Pineda DKM, Rosas GG, Rosas GMI, Rosas UA. 2008. Biopsias de médula ósea: utilidad y limitaciones. Patología. 46(1):237-47.

Puie N, Trica LP, Naggy GA, Rusu I, Sovrea AS, Georgiu C. 2017. Hemorrhagic polypoid gastric and colonic metastasis nine years after uterine leiomyosarcoma, case report. Romanian Journal of Morphology and Embryology. 58(3):1017-22.

Ramos-Vara JA. 2005. Technical aspects of immunohistochemistry. Veterinary Pathology. 41 (4):405-26. doi: 10.1354/vp.42-4-405

Reagan WJ, Irizarry-Rovira A, Poitout-Belissent F, Provencher BA, Shashi K, Travlos G, Walker D, Bounous D, Walter G. 2011. Best practices for evaluation of bone marrow in nonclinical toxicity studies. Toxicologic Pathology. 30(2):435-48.

doi: 10.1177/0192623310396907

Sanpera N, Masot N, Janer M, Romeo C, De Pedro R. 2002. Oestrogen-induced bone marrow aplasia in a dog with a Sertoli cell tumour. Journal of Small Animal Practice. 43(8):365-9.

Serin G, Aydogan A, Yaygingul R, Tuncan R. 2010. Uterine leiomyosarcoma in a dog: a case report. Veterinarni Medicina. 55(8):405-8.

doi: 10.17221/2959-VETMED

Sontas HB, Dokuzeylu B, Turna O, Ekici H. 2009. Estrogen-induced myelotoxicity in dogs: a review. Canadian Veterinary Journal. 50(10):1054-8.

Suh MJ, Park DC. 2008. Leiomyosarcoma of the vagina: a case report and review from the literature. Journal of Gynecologic Oncology. 19(4): 261-4. doi: 10.3802/jgo.2008.19.4.261

Teramae M, Fukuda T, Imai K, Yamauchi M, Hashiguchi Y, Ichimura T, Yasui T, Sumi T. 2014. Leiomyosarcoma of the vulva: a case report. International Journal of Reproduction, Contraception, Obstetrics and Gynecology. 3(1): 225-8. doi: 10.5455/2320-1770.ijrcog20140345

Verin R, Cian F, Stewart J, Binanti D, MacNeill AL, Piviani M, Monti P, Baroni G, Calvez S, Scase TJ, Finotello R. 2018. A clinical, cytologic, histopathologic, immunohistochemical, and ultrastructural study. Veterinary Pathology. 55 (4):5019.

doi: 10.1177/0300985818759772

Weiss DJ, Wardrop JK. 2010. Schalm's Veterinary Hematology. $6^{\circ}$ Ed. Ames, Wiley-Blackwell. 